\title{
Optical effects induced by epitaxial tension in lead titanate
}

A. Dejneka, D. Chvostova, O. Pacherova, T. Kocourek, M. Jelinek, and M. Tyunina

Citation: Appl. Phys. Lett. 112, 031111 (2018);

View online: https://doi.org/10.1063/1.5013640

View Table of Contents: http://aip.scitation.org/toc/apl/112/3

Published by the American Institute of Physics

\section{Articles you may be interested in}

The interplay between excitons and trions in a monolayer of $\mathrm{MoSe}_{2}$

Applied Physics Letters 112, 031107 (2018); 10.1063/1.5019177

Streak camera imaging of single photons at telecom wavelength

Applied Physics Letters 112, 031110 (2018); 10.1063/1.5004110

Broadband nonlinear optical response of monolayer $\mathrm{MoSe}_{2}$ under ultrafast excitation

Applied Physics Letters 112, 031108 (2018); 10.1063/1.5010060

Photo-conductive detection of continuous THz waves via manipulated ultrafast process in nanostructures Applied Physics Letters 112, 031102 (2018); 10.1063/1.5008790

Polarized micro-Raman studies of femtosecond laser written stress-induced optical waveguides in diamond Applied Physics Letters 112, 031109 (2018); 10.1063/1.5017108

Infrared emission of a freestanding plasmonic membrane

Applied Physics Letters 112, 031104 (2018); 10.1063/1.5017194

\section{Scilight}

Sharp, quick summaries illuminating the latest physics research

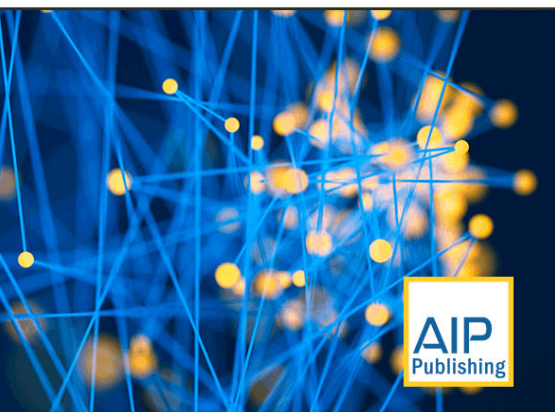




\title{
Optical effects induced by epitaxial tension in lead titanate
}

\author{
A. Dejneka, ${ }^{1}$ D. Chvostova, ${ }^{1}$ O. Pacherova, ${ }^{1}$ T. Kocourek, ${ }^{1}$ M. Jelinek, ${ }^{1}$ and M. Tyunina ${ }^{1,2, a)}$ \\ ${ }^{1}$ Institute of Physics of the Czech Academy of Sciences, Na Slovance 2, 18221 Prague, Czech Republic \\ ${ }^{2}$ Microelectronics Research Unit, Faculty of Information Technology and Electrical Engineering, \\ University of Oulu, P. O. Box 4500, FI-90014 Oulu, Finland
}

(Received 14 November 2017; accepted 6 January 2018; published online 19 January 2018)

\begin{abstract}
Single-crystal-type epitaxial films of perovskite oxide ferroelectrics are attractive for integrated photonic applications because of the remarkable optical properties and effects in ferroelectrics. The properties of the films may be influenced by epitaxial strain arising from the film-substrate mismatch. Here, dramatic strain-induced changes of the absorption and refraction are experimentally detected by spectroscopic ellipsometry in epitaxial films of archetypical ferroelectric $\mathrm{PbTiO}_{3}$. Comparison of the properties of a tensile-strained film with those of reference films and crystals reveals that epitaxial tension produces blueshifts of the primary above-bandgap absorption peaks by $1 \mathrm{eV}$ and a decrease in the refractive index by 0.5 in the transparent spectral range. The obtained quadratic electrooptic and effective elastooptic coefficients exceed the bulk values by orders of magnitude. The experimental observations prove that epitaxy is a powerful tool for engineering unprecedented optical properties that may enable future photonics innovations. Published by AIP Publishing. https://doi.org/10.1063/1.5013640
\end{abstract}

Lead titanate $\left(\mathrm{PbTiO}_{3}\right.$ or PTO further) is an archetypical representative of perovskite oxide ferroelectrics. ${ }^{1}$ This family of materials is known for numerous strong effects and unique response functions, among which linear and nonlinear electro-, elasto-, and piezo-optic effects are especially attractive for photonic applications. ${ }^{1-3}$ The optical properties of PTO were also found to be promising for applications, ${ }^{4}$ which are hindered by difficulties in the synthesis of sufficiently large high-quality crystals and their integration. Great progress in growth of single-crystal-type epitaxial ferroelectric films removes these obstacles and, concurrently, raises questions on the effects of epitaxy on the optical properties of ferroelectrics in general and of PTO in particular. Because of mismatches in crystal lattices and thermal expansion coefficients between the films and substrate materials, epitaxial films may experience lattice strain. In this letter, we report dramatic changes in the absorption coefficient and refractive index, which are induced by epitaxial tensile strain in PTO. We experimentally detect these changes using spectroscopic ellipsometry studies and by comparing the properties of a tensile-strained film with those of reference polydomain single crystals and films and epitaxial films under minor compression. We show that epitaxial tension produces significant blueshifts of the main absorption peaks and a huge decrease in the refraction index in the transparency spectral range. We discuss our observations in terms of the existing models, none of which appear adequate to explain our observations.

Thin films of perovskite ferroelectric PTO were grown by pulsed laser deposition using (001) $\mathrm{SrTiO}_{3}$ (STO) and (011) $\mathrm{DyScO}_{3}$ (DSO) single-crystal substrates (MTI Corp.). A theoretical biaxial in-plane (parallel to the substrate surface) strain is defined here as $s=\left(a_{S} / a_{P T O}-1\right)$, where $a_{S}$ and $a_{P T O}$ are the lattice parameters of the substrate and

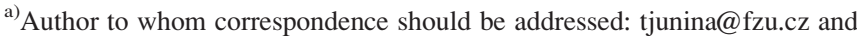
marina.tjunina@oulu.fi
}

unstressed PTO. Because PTO experiences the cubic-totetragonal phase transition at $760 \mathrm{~K}$ on cooling, the parameter $a_{P T O}$ is that of the cubic cell at high temperatures and that $a_{P T O}=b_{P T O}$ of the tetragonal cell at low temperatures. ${ }^{5,6} \mathrm{At}$ the deposition temperature of $973 \mathrm{~K}$, the strain is compressive, $-1.4 \%$, in the PTO films on STO and weakly tensile, $0.1 \%$, in the films on DSO. On cooling to room temperature, the theoretical misfit strain decreases to nearly zero in the PTO/STO film and reaches $1.4 \%$ in the PTO/DSO film. Additionally, a mismatch between thermal expansion coefficients of the substrates and films can affect the room temperature strain state in the films. As the elastic energy of strained films increases with the increasing film thickness, the relaxation of misfit strain starts and pseudomorphic growth terminates at a critical thickness. ${ }^{7}$ To obtain pseudomorphic $c$-type epitaxial PTO films, which possess an out-of-plane direction of polarization and are free of $a$-domains possessing in-plane polarization, the small thickness of approximately $8 \mathrm{~nm}$ was selected for the PTO films on both STO and DSO. ${ }^{8,9}$ We note that the surface cell of the (011)DSO is nearly quadratic, allowing for neglecting in-plane anisotropy in the films. ${ }^{8,9}$ A polydomain film of $\sim 400 \mathrm{~nm}$ in thickness, as determined using an Alpha-Step 500 profilometer, was grown on STO for comparison. ${ }^{10}$

The crystal structure of the films was studied by highresolution X-ray diffraction (XRD) on a Bruker D8 DISCOVER SUPER SPEED SOLUTION diffractometer using $\mathrm{Cu} \mathrm{K} \alpha$ radiation from a rotating anode tube. The $\Theta-2 \Theta$ scans in the range of $2 \Theta=\left(10^{\circ}-130^{\circ}\right)$ and reciprocal space maps in the vicinity of the perovskite (103) and (002) reciprocal lattice points were acquired. The in-plane (parallel to the substrate surface) and out-of-plane (normal to the substrate surface) lattice parameters were estimated from the positions of diffractions using LEPTOS software and taking the substrates as a reference. The analysis revealed growth of 
perovskite films with the $(00 l)$ planes parallel to the substrates' surface (Fig. 1). The presence of Laue satellites indicates the high crystal and surface quality of the 8-nm-thick films [Figs. 1(b) and 1(d)]. The thickness of each film was determined from the positions of the satellites: $(7.5 \pm 0.1) \mathrm{nm}$ of the PTO/STO film and $(7.8 \pm 0.1) \mathrm{nm}$ of the PTO/DSO film. The reciprocal space investigations showed the cubeon-cube-type epitaxial relationships PTO[100](001)||STO [100](001) and PTO[100](001)||DSTO[110](011) and the absence of $a$-domains in these films. The films are coherent and pseudomorphic to the substrates, in agreement with the previous reports. ${ }^{8-10}$ The crystal structure of the films can be interpreted as tetragonal, with the shorter in-plane lattice parameters $a_{f}$ equal to those of the substrates (see supplementary material for more XRD details). The measured out-ofplane lattice parameters $c_{f}$ are $0.417 \mathrm{~nm}$ and $0.405 \mathrm{~nm}$ in the films on STO and DSO, respectively. Compared to tetragonal bulk PTO, ${ }^{5,6}$ the film on STO is weakly elongated in the outof-plane direction. The PTO/DSO film experiences in-plane biaxial tension and out-of-plane compression. The tetragonality $c f / a_{f}$ is approximately 1.06 in the PTO/STO film and is smaller, 1.02, in the PTO/DSO film. The calculated ferroelectric polarization is out-of-plane in both films, and its magnitude differs slightly: $0.92 \mathrm{C} / \mathrm{m}^{2}$ in the PTO/STO film and $0.80 \mathrm{C} / \mathrm{m}^{2}$ in the PTO/DSO film compared to $0.87 \mathrm{C} / \mathrm{cm}^{2}$ in bulk. ${ }^{11}$

The effects of epitaxial tension on the optical properties of PTO are revealed here by comparing the properties of the 8-nm-thick PTO/DSO film with those of the PTO/STO film. Additionally, a 400-nm-thick polydomain PTO/STO film, containing both the $c$ - and $a$-type domains, ${ }^{10}$ and a polydomain PTO bulk crystal are used as references for comparison.

The optical properties of the films, crystal, and substrates were studied using variable angle spectroscopic ellipsometry, which is an ideal and precise measurement technique for determining optical and hence physical properties of materials at the nanoscale. ${ }^{12}$ The bare STO and DSO
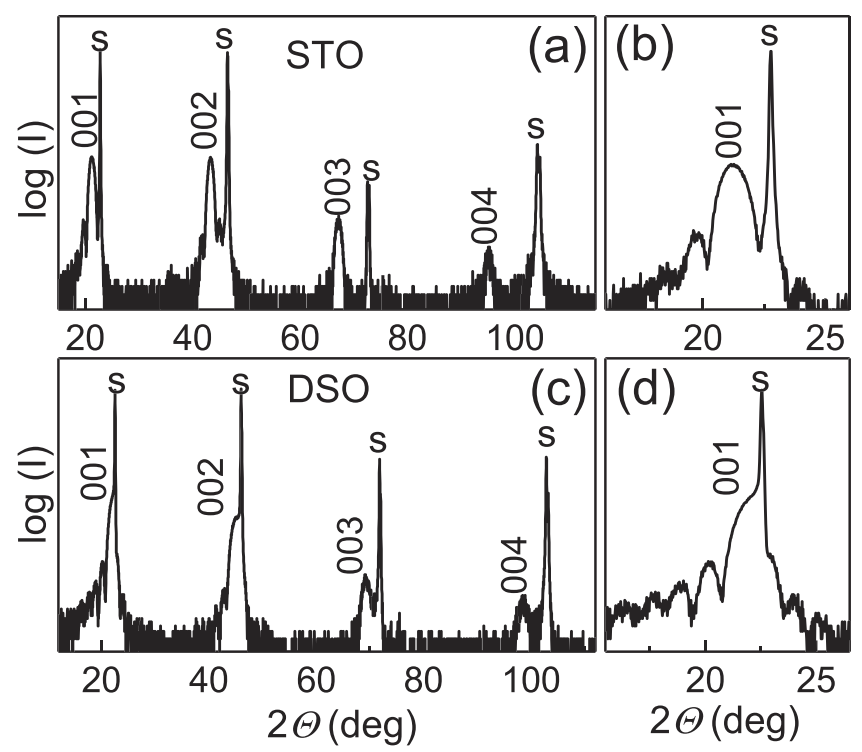

FIG. 1. X-ray diffraction $\Theta-2 \Theta$ scans in the 8 -nm-thick PTO films on (a) and (b) STO and (c) and (d) DSO. The panels (b) and (d) show details of the scans around the perovskite (001) diffractions. The indices of the perovskite PTO peaks are indicated in the plots. The substrate diffractions are indicated by "s". single-crystal substrates were investigated to ensure accurate studies of the properties of the films. The measurements were performed on a J.A. Woollam VUV ellipsometer at room temperature, in a dry nitrogen atmosphere, and at photon energies of $(0.75-8.8) \mathrm{eV}$. The ellipsometric spectra were acquired at two angles of incidence of $65^{\circ}$ and $70^{\circ}$, with the energy step of $0.02 \mathrm{eV}$, and using averaging over 200-400 optical cycles. The optical constants of the films, crystal, and substrates were extracted from the spectra of ellipsometric angles $\psi$ and $\Delta$ using a commercial WVASE32 software package. ${ }^{13}$ The modeling procedure was initiated by constructing a four-phase model, which includes a semiinfinite substrate, homogeneous film, surface roughness, and ambient gas. The PTO film's thickness extracted from the XRD data was used as a starting parameter. A three-phase model for the crystal and substrates includes a semi-infinite crystal, surface roughness, and ambient gas, respectively. The surface roughness was calculated as a mixture of $50 \%$ solid and $50 \%$ voids, in accordance with the Bruggeman effective medium approximation. ${ }^{14}$ The complex dielectric function was presented in a multi-oscillator form using one Cody-Lorentz oscillator and six Gaussian oscillators. ${ }^{15,16}$ The oscillators' parameters and the layer thicknesses were fitting variables and determined simultaneously over the entire spectral range using the Levenberg-Marquardt algorithm. ${ }^{17}$ Because of strong correlation between the thickness and optical constants of thin films (thickness $<10 \mathrm{~nm}$ ), ${ }^{18}$ the thickness was varied around that determined from XRD data in the range of $(8 \pm 1) \mathrm{nm}$ in the 8 -nm-thick films. The $(\psi, \Delta)$ spectra were fit using the weighted error function. The roughness layer thickness was $(0.2 \pm 0.1)$ and $(4.04 \pm 0.02)$ $\mathrm{nm}$ in the PTO films and crystal, respectively. Direct numerical inversion was used to solve for the dielectric function, with the obtained film thickness and surface roughness being fixed. ${ }^{17,19}$ This approach ensures high precision for determining the dielectric function in the spectral regions, where absorption is weak. ${ }^{19}$ The absorption coefficient $\alpha$ and index of refraction $n$ were calculated from the dielectric functions obtained by numerical inversion. Examples of the fits are shown in the supplementary material.

We note that isotropic optical property models are valid for the PTO crystal and the 400-nm-thick film because of their polydomain microstructures. Also, only a minor anisotropy was detected in a single-domain tetragonal PTO crystal, ${ }^{20}$ which justifies isotropic models for the 8-nm-thick films. Experimentally detected off-diagonal elements of the Jones matrix are nearly zero over the entire spectral range from $0.74 \mathrm{eV}$ to $8.8 \mathrm{eV}$, supporting the validity of the isotropic model here.

The absorption spectra of the films and the crystal are shown in Fig. 2. An onset of absorption $\left(\alpha=10^{4} \mathrm{~cm}^{-1}\right)$ is observed at approximately $3.4 \mathrm{eV}$ in all PTO samples. The absorption edges are similar in the polydomain PTO/STO film and in the crystal [Fig. 2(b)], with the thin PTO/STO film exhibiting a minor redshift of the edge [Fig. 2(a)]. The spectra are dominated by four peaks at the energies of $\sim 4.4 \mathrm{eV}$ (A), $\sim 5.5 \mathrm{eV}(\mathrm{B}), \sim 7.5 \mathrm{eV}(\mathrm{C})$, and $\sim 8.6 \mathrm{eV}$ (D) in the PTO/STO films and the PTO crystal [Figs. 2(a) and 2(b)]. Compared to the reference polydomain film and crystal, the magnitudes of the $\mathrm{B}, \mathrm{C}$, and $\mathrm{D}$ peaks are somewhat lowered in the $c$-type 

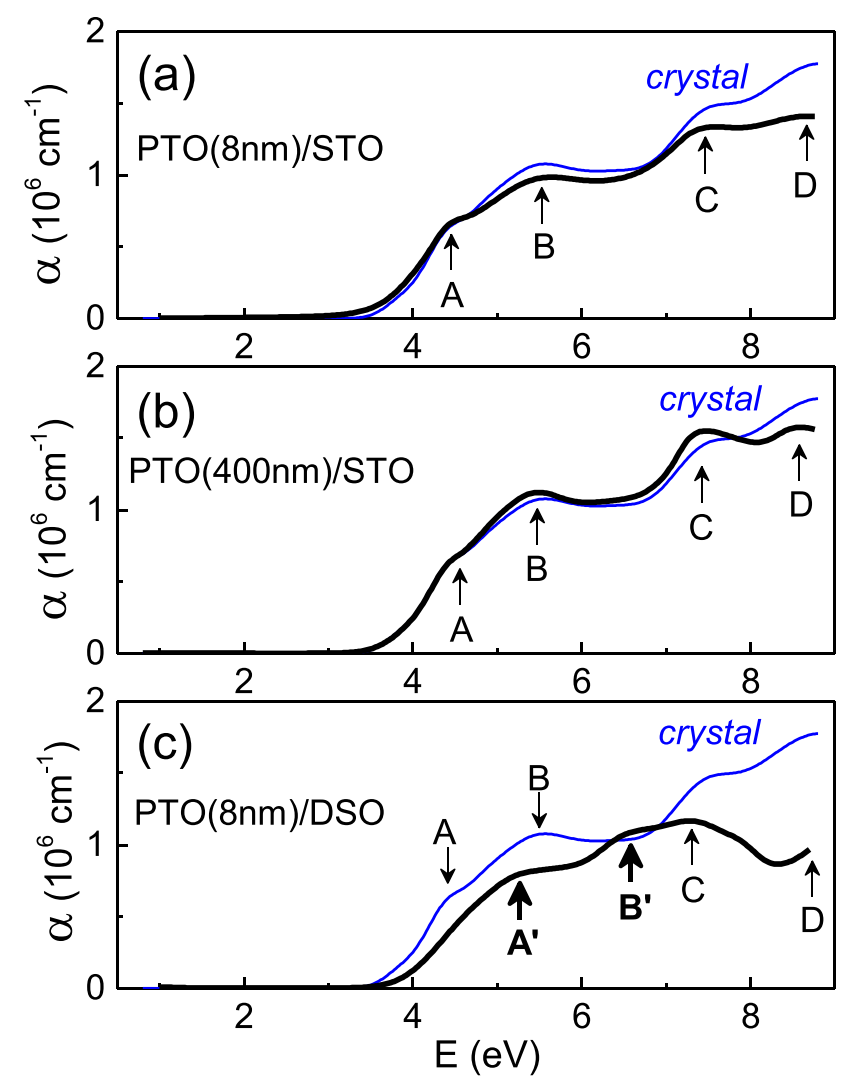

FIG. 2. Absorption coefficient $\alpha$ as a function of photon energy in the PTO films (thick curves) compared to the crystal (thin curves). The films of (a) and (c) $8 \mathrm{~nm}$ and (b) $400 \mathrm{~nm}$ in thickness are deposited on the (a) and (b) STO and (c) DSO substrates. Arrows indicate the characteristic spectral features.

PTO/STO film. The results in [Figs. 2(a) and 2(b)] suggest that interband optical transitions, manifested by the absorption peaks, are weakly affected by epitaxial growth of the thin $c$ type, practically unstrained PTO film on STO. Likewise, the presence of $c / a$-domains appears to have no significant influence on these transitions in PTO either.

As seen from Fig. 2(c), epitaxial tension dramatically alters the absorption spectrum in the PTO/DSO film compared to the crystal and the PTO/STO films. The changes can be interpreted as massive blueshifts of the peaks $\mathrm{A}^{\prime}$ and $\mathrm{B}^{\prime}$ by almost $1 \mathrm{eV}$ in the PTO/DSO films compared to the A and B peaks in the PTO crystal. The positions of the higherenergy peaks $\mathrm{C}$ and $\mathrm{D}$ are approximately the same everywhere although their magnitudes are suppressed in the PTO/ DSO film.

A closer inspection of the absorption edges was performed using Tauc plots (Fig. 3). ${ }^{21}$ Indirect bandgaps ${ }^{22}$ are detected in the crystal and all films. The bandgap energy $E_{g}$ extracted from the linear fits $\left[(\alpha E)^{1 / 2} \propto\left(E-E_{g}\right)\right]$ is $(3.3 \pm 0.05) \mathrm{eV}$ in both the crystal and the 400 -nm-thick PTO/STO film [Fig. 3(a)]. The bandgap is $3.4 \mathrm{eV} \pm 0.05$, i.e., practically similar to that in the crystal, in the 8-nm-thick PTO/DSO film [Fig. 3(b)]. Thus, epitaxial tension produces the giant $\mathrm{A}-\mathrm{A}^{\prime}$ and $\mathrm{B}-\mathrm{B}^{\prime}$ blueshifts, while the bandgap increase is minor. A smaller bandgap of $(3.1 \pm 0.05) \mathrm{eV}$ is obtained in the 8-nm-thick PTO/STO film [Fig. 3(b)].

Our experimental observations point to complex epitaxy-induced changes in the optical band-to-band

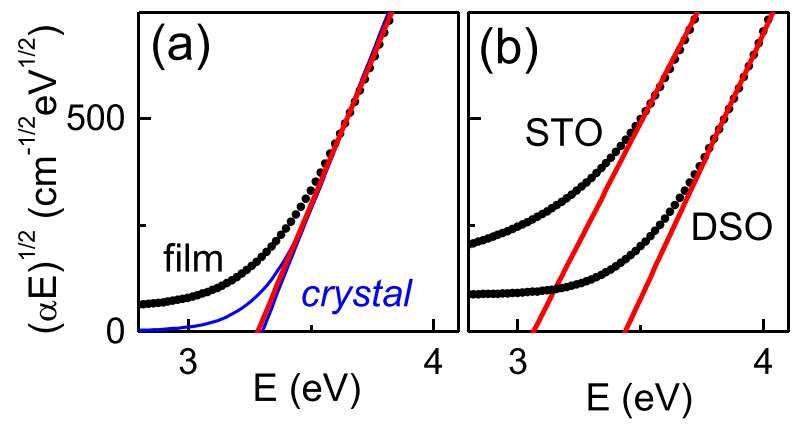

FIG. 3. Tauc plots for indirect bandgaps in the (a) PTO crystal and 400-nmthick PTO/STO film and (b) 8-nm-thick PTO/STO and PTO/DSO films. Straight lines show fits to $\left[(\alpha E)^{1 / 2} \propto\left(E-E_{g}\right)\right]$.

transitions in PTO, which are not accounted for by the state-of-the-art theoretical modeling. In particular, the firstprinciples analysis of epitaxial perovskite pseudo-cubic films predicts that biaxial in-plane strain should lead to a relative lowering of the valence band and, consequently, to a bandgap narrowing. ${ }^{23,24}$ This tendency should be independent of the sign of the strain, tensile or compressive. Concurrently, strain-induced ferroelectric polarization should raise the valence band and lead to a widening of the bandgap. ${ }^{23-25}$ Our observations suggest that the effect of weak compressive strain may dominate over that of strain-induced polarization, leading to bandgap narrowing in the 8-nm-thick PTO/STO film. However, the bandgap increase in the 8-nm-thick PTO/ DSO film contradicts the predicted tendencies: the tensile strain, smaller tetragonality, and, consequently, smaller polarization are expected to induce bandgap narrowing, in contrast to the observations. In an attempt to resolve this discrepancy, we assume that the PTO/DSO film may be located close to a theoretical boundary between the tetragonal and monoclinic-type $\mathrm{Cm}$ phases. ${ }^{11}$ The $\mathrm{Cm}$ phase is characterized by a rotation of ferroelectric polarization away from the strict out-of-plane direction. Such polarization rotation was, indeed, detected by sophisticated synchrotron XRD analysis of the ultrathin PTO/DSO films. ${ }^{8}$ Although the occurrence of the $\mathrm{Cm}$ phase is impossible to reveal using the laboratory $\mathrm{XRD}$ studies, the polarization rotation may cause changes in the optical properties of the PTO/DSO film. To corroborate the role of the $\mathrm{Cm}$ phase, we inspected the refractive index $n$ in the transparency range of PTO. According to the modeling in Ref. 11, the $\mathrm{Cm}$ phase should exhibit an increase in $n$ compared to that in the PTO crystal. However, our measurements show an immense decrease in the index in the PTO/DSO film, in contrast to the calculations (Fig. 4). Thus, the optical properties of the tensile strained PTO/DSO film cannot be explained by polarization rotation.

According to the well-established semi-empirical model, a decrease in $n$ is related to a blueshift $\Delta E_{A}$ of the main absorption peak in perovskite-type ferroelectrics ${ }^{26}$

$$
\begin{gathered}
\Delta\left(\frac{1}{n^{2}}\right) \approx g \Delta\left(P^{2}\right), \\
\Delta E_{A} \approx 20 g \Delta P,
\end{gathered}
$$

where $g$ is the quadratic electro-optic coefficient, $P$ is the ferroelectric polarization, and the tensor form of relationships 


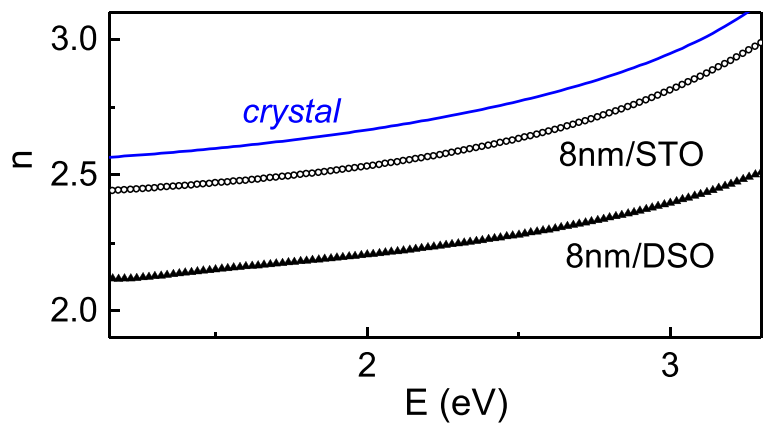

FIG. 4. Index of refraction $n$ as a function of photon energy in the transparency range in the PTO crystal (thin curve), 8-nm-thick PTO/STO film (open symbols), and 8-nm-thick PTO/DSO film (solid symbols).

is omitted for simplicity. Using the refractive indices, measured at the photon energies from $1 \mathrm{eV}$ to $3.3 \mathrm{eV}$, and positions of the $\mathrm{A}\left(\mathrm{A}^{\prime}\right)$ peaks in the films and the crystal, and considering the theoretical polarization therein, we estimate the coefficient $g$ from expression (1). The coefficient is $g_{\text {PTO/STO }} \approx(0.12-0.18) \mathrm{m}^{4} / \mathrm{C}^{2}$ in the PTO/STO film, which is close to the value in ferroelectrics such as $\mathrm{BaTiO}_{3}$ but significantly exceeds that in bulk PTO., ${ }^{4,27}$ For the estimated $g_{\text {PTO/STO, the PTO/STO film should exhibit an approximately }}$ $0.1-\mathrm{eV}$ blueshift of the main absorption peak compared to the crystal. The expected shift agrees with the detected positions of the A and B peaks [Fig. 2(a)]. Also, the decrease in $n$ and the $\mathrm{A}-\mathrm{A}^{\prime}$ and $\mathrm{B}-\mathrm{B}^{\prime}$ blueshifts observed in the PTO/DSO film are in a qualitative agreement with relationships (1-2). However, in contrast to the self-consistent estimations in the $\mathrm{PTO} / \mathrm{STO}$ film, the giant negative coefficient $g_{\mathrm{PTO} / \mathrm{DSO}}$ $\approx-(26-33) \mathrm{m}^{4} / \mathrm{C}^{2}$ is obtained using expression (1), resulting in an extremely large A- $\mathrm{A}^{\prime}$ blueshift calculated using expression (2) in the PTO/DSO film. Thus, the semi-empirical model appears to be insufficient for explaining the optical behavior of PTO under epitaxial tension.

Next, using the refractive index in the PTO/DSO film and the PTO crystal and considering the out-of-plane compressive strain $s_{c} \approx-2.6 \%$ in the PTO/DSO film, we estimate the effective elastooptic coefficient $p_{s}$ in this film ${ }^{11}$

$$
\Delta\left(\frac{1}{n^{2}}\right)=p_{s} s_{c}
$$

The obtained coefficient is $p_{\mathrm{s}} \approx(2.7-2.2)$ in the spectral range of (1.0-3.3) eV. This large value of $p_{s}$ exceeds the typical elastooptic coefficients of (0.1-0.4) in ferroelectrics and those predicted in epitaxial PTO films by approximately an order of magnitude. ${ }^{4,11,26}$ However, note that the gigantic coefficients $p_{s}$ were measured in strained epitaxial films of $\mathrm{BaTiO}_{3}\left(p_{s} \approx 5\right)$ and $\mathrm{K}_{0.5} \mathrm{Na}_{0.5} \mathrm{NbO}_{3}\left(p_{s} \approx 15\right)$ but not $\mathrm{SrTiO}_{3}$ and $\mathrm{NaNbO}_{3}{ }^{28}$ A peculiar contribution of polar phonons to the refractive index was suggested as a possible qualitative explanation. ${ }^{29}$ This contribution and its dependence on epitaxial strain are not considered in theoretical modeling so far.

The immense blueshift of the main absorption peaks, the huge decrease in the refractive index in the visible range, the giant negative quadratic electro-optic coefficient, and the large effective elastooptic coefficient are thus produced by epitaxial in-plane tensile strain in the PTO/DSO film. These dramatic changes are beyond the existing first-principles and semi-empirical models. We stress that many of the previously observed epitaxy-induced changes in the optical properties in ferroelectrics point to insufficient fundamental understanding of these properties. ${ }^{30-34}$ Because epitaxy proves to be a remarkably powerful tool for engineering these properties, more experimental and theoretical investigations in the field are highly desirable.

The results of our work imply that the optical properties of $\mathrm{PbTiO}_{3}$-based perovskite ferroelectrics can be efficiently tuned by growing epitaxial films of these materials. The $\mathrm{PbTiO}_{3}$ and $\mathrm{PbTiO}_{3}$-based [e.g., $\mathrm{Pb}(\mathrm{Zr}, \mathrm{Ti}) \mathrm{O}_{3}$ ] epitaxial films are now successfully deposited on large-area industrial substrates, ensuring especially high potential for applications. Such applications as capacitors and piezoelectric devices are already commercialized. Knowledge-based control of the films' optical properties would allow implementation of photonics applications within the same technological platform. This prospective makes experimental studies of correlation between the microstructure and optical properties highly relevant and important. Concurrently, such studies deliver a valuable input for theoretical analysis aiming at better fundamental understanding of perovskite optics.

In summary, the optical absorption in the spectral range of $(0.8-8.7) \mathrm{eV}$ and refraction in the visible range of (1.0-3.3) eV are experimentally investigated using spectroscopic ellipsometry in the cube-on-cube-type epitaxial perovskite PTO films on the (001) STO and (011) DSO substrates. Compared to the PTO crystal, the in-plane epitaxial tension of $1.4 \%$ was found to produce a blueshift of the main absorption peaks of $1 \mathrm{eV}$ and a decrease of the refraction index by 0.5 . The existing models are unable to account for these dramatic changes.

See supplementary material for XRD data and ellipsometric data and fits.

The authors acknowledge support from the Grant Agency of Czech Republic (Grant Nos. 15-13778S and 1515123S) and Infotech Oulu of the University of Oulu.

\footnotetext{
${ }^{1}$ M. E. Lines and A. M. Glass, Principles and Applications of Ferroelectrics and Related Materials (Clarendon Press, Oxford, 2004).

${ }^{2}$ Ferroelectric Crystals for Photonic Applications: Including Nanoscale Fabrication and Characterization Techniques, edited by P. Ferraro, S. Grilli, and P. De Natale (Springer, Berlin, 2009).

${ }^{3}$ R. W. Boyd, Nonlinear Optics (Academic Press, Oxford, 2008).

${ }^{4}$ M. D. Fontana, F. Abdi, and K. Wojcik, J. Appl. Phys. 77, 2102 (1995).

${ }^{5}$ L. Bornstein, Numerical Data and Functional Relationships in Science and Technology, New Series, Group III, Crystal and Solid State Physics, edited by K. H. Hellwege and A. M. Hellwege (Springer, Berlin, 1981).

${ }^{6}$ S. A. Mabud and A. M. Glazer, J. Appl. Cryst. 12, 49-53 (1979).

${ }^{7}$ M. Ohring, The Materials Science of Thin Films (Academic Press, San Diego, 1992).

${ }^{8}$ G. Catalan, A. Janssens, G. Rispens, S. Csiszar, O. Seeck, G. Rijnders, D. H. A. Blank, and B. Noheda, Phys. Rev. Lett. 96, 127602 (2006).

${ }^{9}$ O. Nesterov, S. Matzen, C. Magen, A. H. G. Vlooswijk, G. Catalan, and B. Noheda, Appl. Phys. Lett. 103, 142901 (2013).

${ }^{10}$ J. Peräntie, M. S. Stratulat, J. Hannu, H. Jantunen, and M. Tyunina, APL Mater. 4, 016104 (2016).

${ }^{11}$ L. Chen, Y. Yang, Z. Gui, D. Sando, M. Bibes, X. K. Meng, and L.
} Bellaiche, Phys. Rev. Lett. 115, 267602 (2015). 
${ }^{12}$ M. Schubert, Ann. Phys. 15, 480 (2006).

${ }^{13}$ Guide to Using WVASE 32: Spectroscopic Ellipsometry Data Acquisition and Analysis Software (J. A. Woollam, Inc., 2008).

${ }^{14}$ H. Fujiwara, J. Koh, P. I. Rovira, and R. W. Collins, Phys. Rev. B 61, 10832 (2000).

${ }^{15}$ A. S. Ferlauto, G. M. Ferreira, J. M. Pearce, C. R. Wronski, R. W. Collins, X. Deng, and G. Ganguly, J. Appl. Phys. 92, 2424 (2002).

${ }^{16}$ J. Price, P. Y. Hung, T. Rhoad, B. Foran, and A. C. Diebold, Appl. Phys. Lett. 85, 1701 (2004).

${ }^{17}$ J. J. More, "The Levenberg-Marquardt algorithm: implementation and theory," in Numerical Analysis (Springer, 1978), pp. 105-116.

${ }^{18}$ J. N. Hilfiker, N. Singh, T. Tiwald, D. Convey, S. M. Smith, J. H. Baker, and H. G. Tompkins, Thin Solid Films 516, 7979 (2008).

${ }^{19}$ L. K. Gautam, H. Haneef, M. M. Junda, D. B. S. John, and N. J. Podraza, Thin Solid Films 571, 548 (2014).

${ }^{20}$ W. Kleemann, F. J. Schafer, and D. Rytz, Phys. Rev. B 34, 7873 (1986).

${ }^{21}$ J. Tauc, R. Grigorovici, and A. Vancu, Phys. Status Solidi 15, 627 (1966).

${ }^{22}$ S. Piskunov, E. Heifets, R. I. Eglitis, and G. Borstel, Comput. Mater. Sci. 29, 165 (2004).
${ }^{23}$ R. F. Berger, C. J. Fennie, and J. B. Neaton, Phys. Rev. Lett. 107, 146804 (2011).

${ }^{24}$ F. Wang, I. Grinberg, and A. M. Rappe, Appl. Phys. Lett. 104, 152903 (2014).

${ }^{25}$ J. R. Brews, Phys. Rev. Lett. 18, 662 (1967).

${ }^{26}$ M. DiDomenico, Jr. and S. H. Wemple, J. Appl. Phys. 40, 720 (1969).

${ }^{27}$ P. Bernasconi, M. Zgonik, and P. Gunter, J. Appl. Phys. 78, 2651 (1995).

${ }^{28}$ A. Dejneka and M. Tyunina, Adv. Appl. Ceram. 117, 62 (2018).

${ }^{29}$ M. Veithen and P. Ghosez, Phys. Rev. B 71, 132101 (2005).

${ }^{30}$ E. Chernova, O. Pacherova, D. Chvostova, A. Dejneka, T. Kocourek, M. Jelinek, and M. Tyunina, Appl. Phys. Lett. 106, 192903 (2015).

${ }^{31}$ M. Tyunina, D. Chvostova, L. D. Yao, A. Dejneka, T. Kocourek, M. Jelinek, and S. van Dijken, Phys. Rev. B 92, 104101 (2015).

${ }^{32}$ M. Tyunina, L. D. Yao, D. Chvostova, T. Kocourek, M. Jelinek, A. Dejneka, and S. van Dijken, New J. Phys. 17, 043048 (2015).

${ }^{33}$ M. Tyunina, L. Yao, D. Chvostova, A. Dejneka, T. Kocourek, M. Jelinek, V. Trepakov, and S. van Dijken, Sci. Technol. Adv. Mater. 16, 026002 (2015).

${ }^{34}$ A. Dejneka, M. Tyunina, J. Narkilahti, J. Levoska, D. Chvostova, L. Jastrabik, and V. Trepakov, Phys. Solid State 52, 2082 (2010). 